\title{
Radio-Frequency Plasma-Induced Biocompatibility of Polyimide Substrates
}

\author{
Magdaleno R. Vasquez, Jr., ${ }^{a,{ }^{*}}$ Eloise I. Prieto, ${ }^{b}$ \& Motoi Wadac \\ aDepartment of Mining, Metallurgical, and Materials Engineering, College of Engineering, \\ University of the Philippines, Diliman, Quezon City 1101, Philippines; bNational Institute of \\ Molecular Biology and Biotechnology, College of Science, University of the Philippines, Diliman, \\ Quezon City 1101, Philippines; 'Graduate School of Science and Engineering, Doshisha \\ University, Kyotanabe, Kyoto 610-0321, Japan \\ *Address all correspondence to: Magdaleno R. Vasquez, Jr., DMMME Bldg., Velasquez St. cor. C.P. Garcia Ave., UP \\ Diliman, Quezon City 1101, Philippines; Tel.: +63 2981 8500,x3132, E-mail: mrvasquez2@up.edu.ph
}

\begin{abstract}
Surfaces of polyimide (PI) sheets were modified using 13.56-MHz radio-frequency discharges to enhance their affinity with fibroblast cells. Physico-chemical analysis of pristine and plasma-treated PI sheets showed different responses against argon (Ar), oxygen $\left(\mathrm{O}_{2}\right)$, and nitrogen $\left(\mathrm{N}_{2}\right)$ as the plasma process gases. Overall, hydrophilicity of treated PI surfaces was enhanced, and its surface free energy increased from $53 \mathrm{dyn} / \mathrm{cm}$ to at least $73 \mathrm{dyn} /$ $\mathrm{cm}$. Surface roughness values also increased from 1.3 to $40.8 \mathrm{~nm}$, as demonstrated by atomic force microscopy analyses. Infrared spectral analyses showed a decrease of imide functional group peak intensities on plasma exposure, corresponding to chemical surface structural changes. In addition, plasma-treated surfaces significantly increased cell adhesion and proliferation compared to pristine samples. $\mathrm{N}_{2}$-containing plasma exhibited the greatest increase among the test gases, due to the possible inclusion of $\mathrm{N}_{2}$-based functional groups that enhance biochemical affinity of fibroblast cells.
\end{abstract}

KEYWORDS: polyimide, plasma treatment, biocompatibility, biofunctionalization, cell adhesion, cell proliferation

\section{INTRODUCTION}

Polyimides (PIs) are characterized by their superior flexibility, high thermal stability, high chemical resistance, and low dielectric constant. ${ }^{1,2}$ Thus, PIs are useful for industrial applications such as microelectronics ${ }^{3,4}$ that include wire insulation, liners, and substrates. In addition to their excellent physical properties and ability to be sterilized, PIs have been determined to elicit no cytotoxic response and low hemolysis, ${ }^{5}$ making them ideal for biomedical applications. Recent work includes the use of PIs as biosensor encapsulants and substrates for neural implants, ${ }^{6}$ regenerative-medicine applications, ${ }^{7}$ cellculture applications, ${ }^{8}$ and other implantable electronic sensor and stimulation systems. ${ }^{9}$ PIs, although demonstrated to be biocompatible, still lack cell-interactive properties that would make them appropriate for tissue-repair applications. ${ }^{7}$ PI substrates therefore need to be surface-modified to improve cell-adhesion and -proliferation properties. Among the surface treatment techniques, plasma-based treatment has been used to improve biomolecular affinity of polymeric substrates ${ }^{10-12}$ and adhesion of different materials. ${ }^{13}$ This dry process is quick, does not affect the bulk properties of the substrates, and does not pro- 
duce toxic by-products. Plasma discharge provides a mixture of neutrals, ions, electrons, radicals, metastables, and ultraviolet radiation, making it a versatile process for tailoring properties of polymeric materials that are located a few nanometers from the surface. By varying gas composition and operating parameters such as incident power, exposure time, and gas-flow rate, precise surface functionalization and activation can be realized.

The present work explores the biochemical response of pristine and plasma-treated PI substrates. Degreased substrates were subjected to a $13.56-\mathrm{MHz}$ radio-frequency (RF) plasma discharge at subatmospheric pressures using different process gases. After plasma treatment, physical and chemical changes of the substrates were compared to untreated samples. Cell-adhesion and -proliferation properties were also examined using a human fibroblast cell line.

\section{EXPERIMENTALDETAILS}

\section{A. Surface Preparation and Characterization}

Commercially available PI (Kapton, The Nilaco Corp.; Tokyo, Japan), with a thickness of $0.05 \mathrm{~mm}$, was cut into $1-\mathrm{cm}$ squares. The substrates, degreased through subsequent sonication in acetone, ethanol, and water baths for $10 \mathrm{~min}$ each, were blow-dried using nitrogen gas. Immediately after degreasing, the samples were transported in ambient conditions, mounted on a flat glass plate, and placed inside a plasma reactor (Fig. 1), a glass bell jar with a diameter of $165 \mathrm{~mm}$ and height of $170 \mathrm{~mm}$. The chamber was terminated by a flange with an RF-powered electrode that also acted as a sample mount. The distance between electrode and gas outlet was $\sim 55 \mathrm{~mm}$. Details of the plasma system can be found in Cagomoc and Vasquez. ${ }^{14}$ The system was evacuated by a rotary pump to $\sim 8$ $\mathrm{Pa}$ before the working gas was introduced through a needle valve. We used argon (Ar),

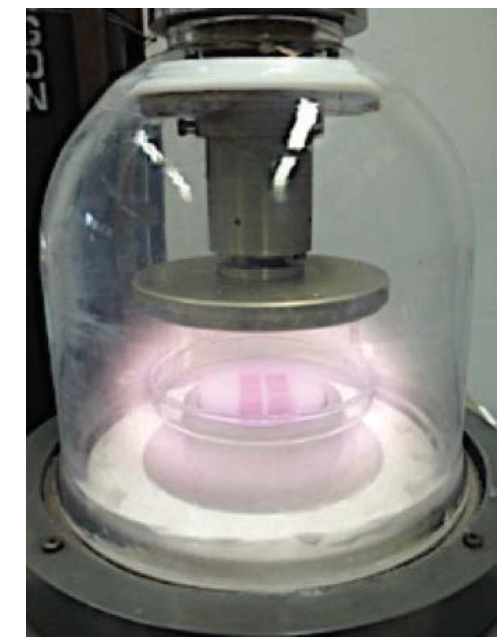

FIG. 1: Image of PI sheets undergoing plasma treatment 
nitrogen $\left(\mathrm{N}_{2}\right)$, and oxygen $\left(\mathrm{O}_{2}\right)$ gases. Operating pressure was maintained at $100 \mathrm{~Pa}$ for all experiments, and exposure time was set to $30 \mathrm{~min}$. Plasma was excited by capacitively coupled 13.56-MHz RF power, with a manual matching circuit that minimized the reflected power. Incident RF power was set to $50 \mathrm{~W}$ for all runs. After plasma treatment, the samples were wrapped in aluminum foil for sterilization, which was conducted in a convection oven (Heratherm, Thermo Fisher Scientific; Waltham, MA) for $3 \mathrm{hr}$ at $180^{\circ} \mathrm{C}$. After sterilization, samples were cooled and prepared for biocompatibility experiments.

We used atomic force microscopy (AFM) for topographic analyses of untreated and plasma-treated PI substrates using XE-Bio AFM (Park Systems; Suwon, Korea) under an intermittent mode. Silicon- and aluminum-coated cantilevers (PPP-NCHR, $10 \mathrm{M}$; Park Systems Inc.; Suwon, Korea) were used that had a typical spring constant of 42 $\mathrm{N} / \mathrm{m}^{-1}$. Surface free energy (SFE) was determined from the Owens-Wendt equation ${ }^{15}$ through contact-angle $(\theta)$ measurements of water (polar) and diiodomethane (nonpolar) test liquids as follows:

$$
\frac{\gamma_{l}(1+\cos \theta)}{2}=\left[\sqrt{\gamma_{l}^{d} \gamma_{s}^{d}}+\sqrt{\gamma_{l}^{p} \gamma_{s}^{p}}\right]
$$

where $\gamma_{1}$ corresponds to surface tension of the liquid; $\gamma_{1}^{d}$ and $\gamma_{1}^{p}$ to dispersive and polar components of liquid surface tension, respectively; and $\gamma_{s}^{d}$ and $\gamma_{s}^{p}$ to dispersive and polar components of solid surface energy. A Fourier-transform infrared (FTIR) spectrophotometer (Shimadzu IR-Prestige 21; Kyoto, Japan) was used to investigate chemical characteristics of the untreated and plasma-treated PI surfaces.

\section{B. Cell Adhesion and Proliferation}

\section{Cell Culture and Seeding of Substrates}

We used human gingival fibroblasts (HGF-1) from the American Type Culture Collection (Rockville, MD). These cells were maintained in Dulbecco's modified Eagle's medium (DMEM; Millipore Sigma; St. Louis, MO) that was supplemented with $10 \%$ fetal bovine serum (FBS; Thermo Fisher Scientific; Waltham, MA) and $0.5 \%$ penicillin-streptomycin (Thermo Fisher Scientific; Waltham, MA) at $37^{\circ} \mathrm{C}$ in a $5 \% \mathrm{CO}_{2}$ humidified incubator. Cells were seeded onto PI substrates inside a 24-well cell-culture plate at 15,000 cells per well. Cell-attachment and proliferation assays were then performed on days 1 and 3 after seeding.

\section{Cell-Attachment Assays}

Cell adhesion on plasma-treated PI substrates was assessed using the LIVE/DEAD Viability/Cytotoxicity Assay Kit (Molecular Probes/Invitrogen; Carlsbad, CA). Substrates were stained with $2 \mu \mathrm{M}$ calcein acetoxymethyl and $4 \mu \mathrm{M}$ ethidium homodimer (EthD-1)

Volume 8, Issue 1, 2018 
Vasquez, Prieto, \& Wada

solution, and the percentage of cell coverage was analyzed by fluorescence micrographs using ImageJ software. ${ }^{16}$ Polystyrene tissue-culture (TC) dishes served as a positive control, and pristine substrates served as a negative control.

\section{Cell-Proliferation Assays}

Cell proliferation on plasma-treated PI substrates was evaluated using PrestoBlue (Molecular Probes/Invitrogen; Carlsbad, CA), a reagent that is reduced by metabolically active cells. The substrates seeded with HGF-1 were incubated with PrestoBlue, after which solution fluorescence was measured with Varioskan Flash (Thermo Fisher Scientific; Waltham, MA) at an excitation and emission wavelength of 560 and $590 \mathrm{~nm}$, respectively. Higher fluorescence values corresponded to greater total metabolic activity. Polystyrene TC dishes served as positive control and pristine substrates as negative control. Obtained fluorescence values were normalized with cells grown on TC dishes.

\section{RESULTS AND DISC USSION}

\section{A. Physic o-Chemical Analyses of Surfaces}

One of the major surface parameters that determine cell-material interaction is surface hydrophilicity. It has been demonstrated that cell-adhesion proteins show better binding to hydrophilic surfaces than to hydrophobic surfaces, leading to more effective cell attachment on hydrophilic materials. ${ }^{17}$ We found that hydrophilicity of PI surfaces significantly improved after plasma treatment, with Ar plasma having a noteworthy increase in hydrophilicity [Fig. 2(a)]. Compared to the $60^{\circ}$ contact angle of the pristine PI surface, treated samples had considerably lower mean contact angles of $10^{\circ}, 11^{\circ}$, and $31^{\circ}$ for $\mathrm{Ar}$, $\mathrm{O}_{2}$, and $\mathrm{N}_{2}$ plasma treatment, respectively. Correspondingly, SFE also increased for all treated substrates [Fig. 2(b)]). Because surface hydrophilicity is influenced by modifications in surface morphology and chemistry, analyses of PI substrates were conducted via AFM and FTIR spectroscopy to determine the effect of plasma treatment on these surface parameters.

Figure 3(a)-(d) show representative $2 \times 2-\mu \mathrm{m}^{2}$ AFM images of pristine and plasmatreated substrates and their corresponding root-mean-square (RMS) roughness. It is evident from the images that plasma treatment of PI substrates resulted in variations in surface morphology. This was confirmed with increased surface roughness, from $1.3 \mathrm{~nm}$ for pristine samples to $9.8,14.6$, and $40.8 \mathrm{~nm}$ for $\mathrm{Ar}, \mathrm{O}_{2}$, and $\mathrm{N}_{2}$ plasma-treated samples, respectively [Fig. 3(e)]. These physical changes may be attributed to plasma energetic particles impinging on the PI substrate, thereby etching its surface. Reactive gases in the discharge such as $\mathrm{O}_{2}$ and $\mathrm{N}_{2}$ also contributed to the increase in roughness through attachment of functional groups on the surface.

FTIR spectral analyses of pristine and treated samples are shown in Fig. 4 (FTIR spectrum images). Figure 4(a) shows typical IR absorption spectra of pristine and plasma-modified PI surfaces. Imide functional groups are apparent at $1780 \mathrm{~cm}^{-1}(C=O$ 

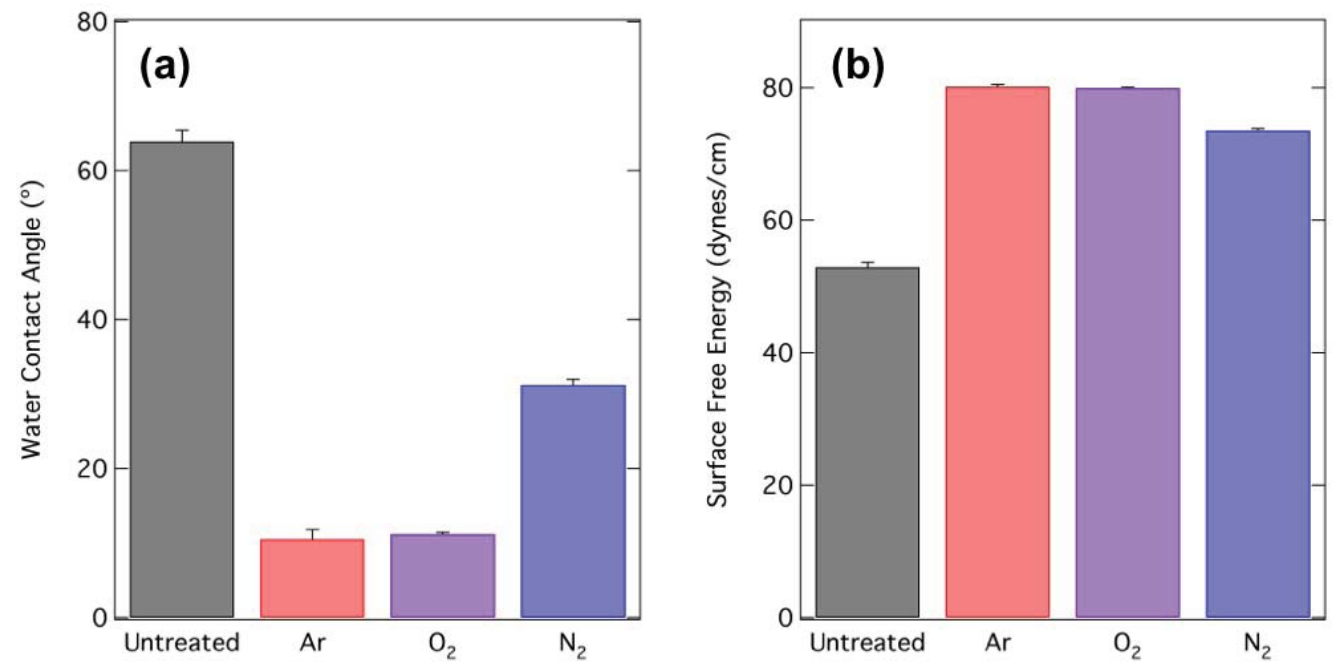

FIG. 2: (a) Water contact angles and (b) SFEs of pristine and plasma-treated substrates
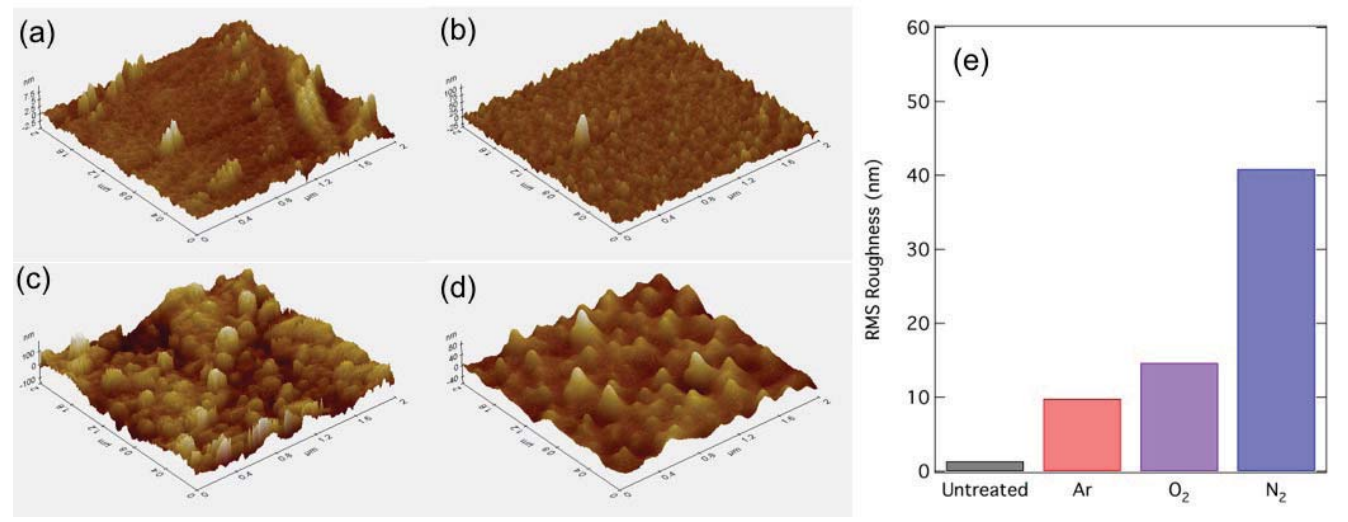

FIG. 3: Morphologies of pristine (a) and plasma-treated samples of (b) $\mathrm{Ar}$, (c) $\mathrm{N}_{2}$, and (d) $\mathrm{O}_{2}$. (e) Comparison of RMS roughness

asymmetrical stretch), $1715 \mathrm{~cm}^{-1}$ (C=O symmetrical stretch), $1375 \mathrm{~cm}^{-1}$ (C-N stretch), and $720 \mathrm{~cm}^{-1}(\mathrm{C}=\mathrm{O}$ bending $)$. We used these peaks that were relevant to the chemical structure of PIs to evaluate changes in IR peaks of plasma-treated surfaces. After reviewing the difference in FTIR spectra of plasma-treated samples [Fig. 4(b)], we observed a decrease in intensity of the imide functional groups. This decrease indicates changes in surface chemical properties such as the reduction of imide functional groups, although the degree of chemical change is challenging to determine from the spectra. This difficulty in detecting chemical changes on PI surfaces may be due to the very thin layers of chemical modifications formed on the substrate after plasma treatment. In addition, the number of detected signals using FTIR spectroscopy was less than the actual amount

Volume 8, Issue 1, 2018 

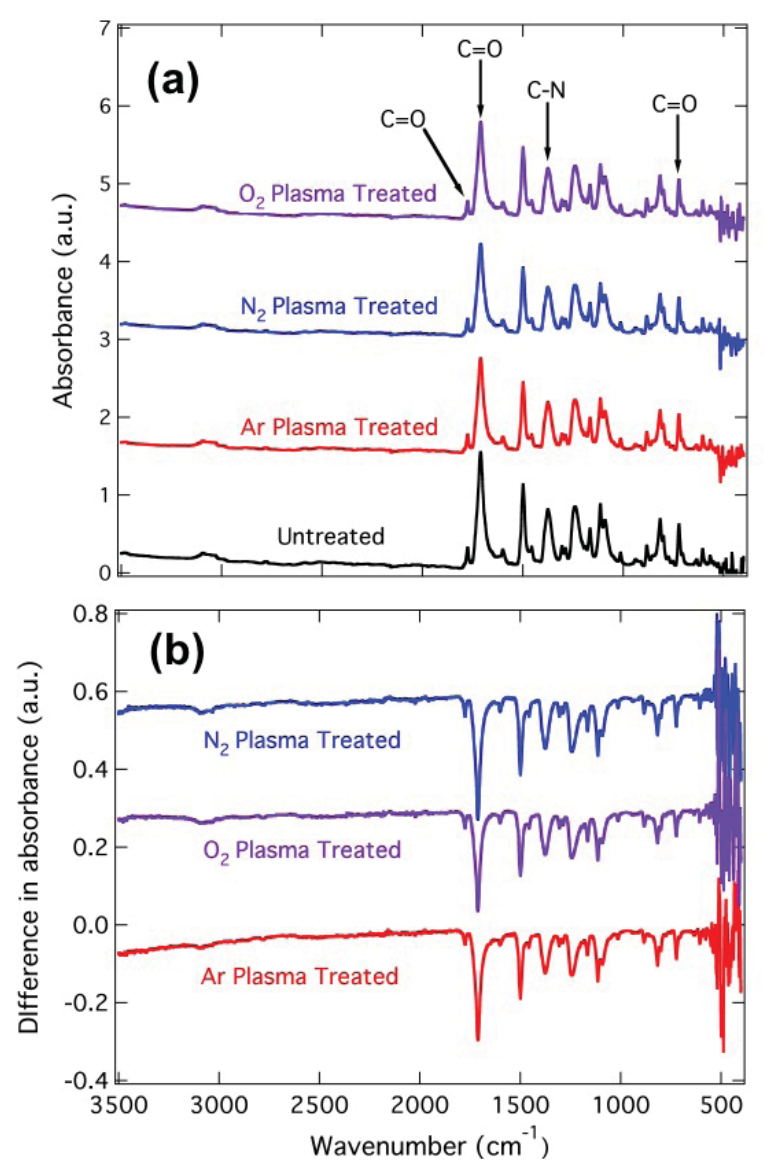

FIG. 4: (a) Typical FTIR spectra of pristine and plasma-modified PI samples; (b) difference in FTIR absorbance of plasma-treated samples

(detection of chemical changes may be improved with X-ray photoelectron spectroscopy). Although FTIR spectral analyses were unable to identify additional functional groups on the treated surfaces, attachment of functional groups on the PI surfaces cannot be discounted, because reactive species are present in the $\mathrm{N}_{2}$ and $\mathrm{O}_{2}$ plasma.

\section{B. Biocompatibility Assays}

Effects of PI surface plasma treatment on cell behavior were investigated for $3 \mathrm{~d}$ using fibroblast cells. Figure 5 shows cells grown on PI surfaces, and analyses of cell morphology revealed the absence of any significant morphological changes between cells grown on pristine and plasma-treated PI. Moreover, their morphologies were similar to cells grown on TC dishes, demonstrating that plasma treatment of PI is not cytotoxic to fibroblast cells. Quantification of cell adhesion in Fig. 5 demonstrated better cell cover- 


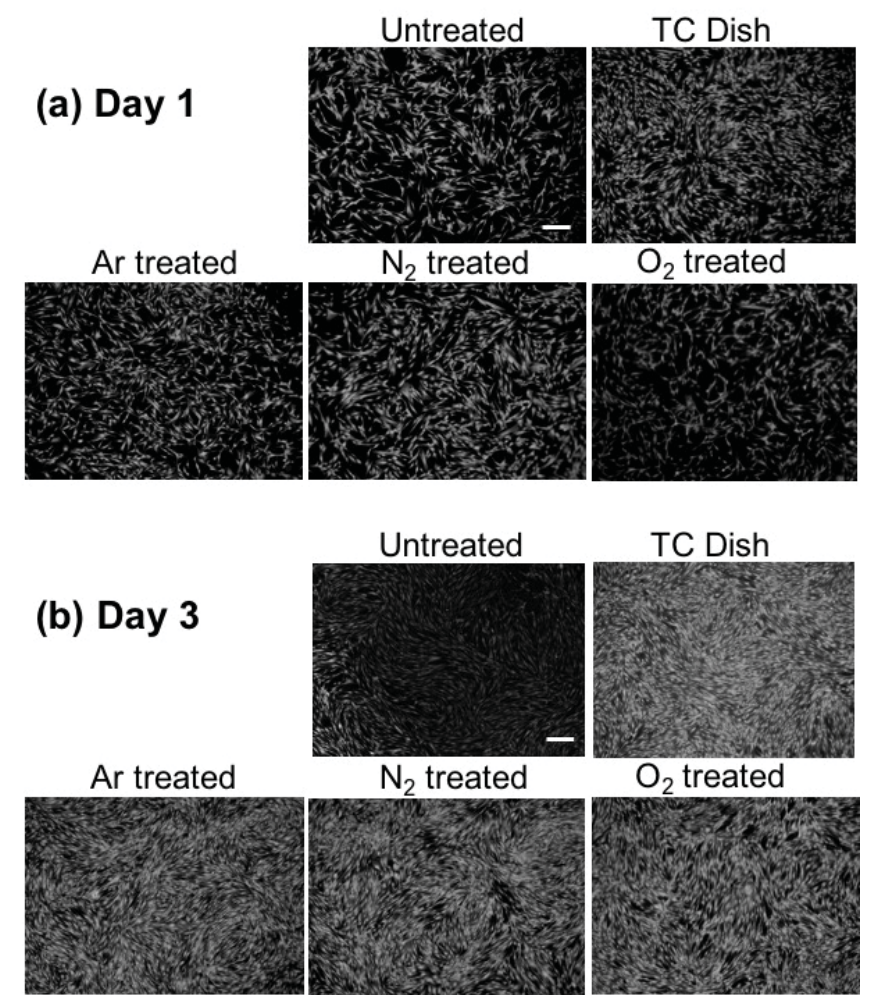

FIG. 5: Representative cell-adhesion profiles of fibroblast cells on pristine and plasma-treated PI surfaces after 1 and $3 \mathrm{~d}$ in culture. Cells were stained with calcein and visualized with fluorescence microscopy. TC, Tissue culture. (Bar, $200 \mu \mathrm{m}$.)

age for plasma-treated PI substrates than for pristine samples. Initial attachment significantly improved with $\mathrm{N}_{2}$ plasma treatment, as revealed by increased cell coverage at day 1 [Fig. 6(a)]. Cell coverage increased for all plasma-treated substrates compared to cells grown on TC dishes, indicating that plasma modification of the PI surface improved cell attachment [Fig. 6(b)].

Figure 7 depicts cell viability on PI substrates relative to untreated controls after 1 and $3 \mathrm{~d}$ of incubation. Although the amounts of viable cells on plasma-treated substrates were initially comparable to those of untreated PIs with only the TC dish showing high viability, significant increase in viability between the treated and pristine samples was apparent at day 3 , indicating that plasma treatment of PI positively influences cell proliferation.

The enhanced initial attachment of fibroblast cells on $\mathrm{N}_{2}$ plasma-treated PI may be due to its hydrophilic nature brought about by its high RMS roughness and the presence of polar groups on the PI surface. $\mathrm{N}_{2}$-containing plasma may include the attachment of amine-based functional groups. Amine functionalities provide positive charges that encourage cell adhesion under physiological conditions. ${ }^{18}$ PI substrates treated with Ar plasma would have surface modifications associated mainly with the etching process as 


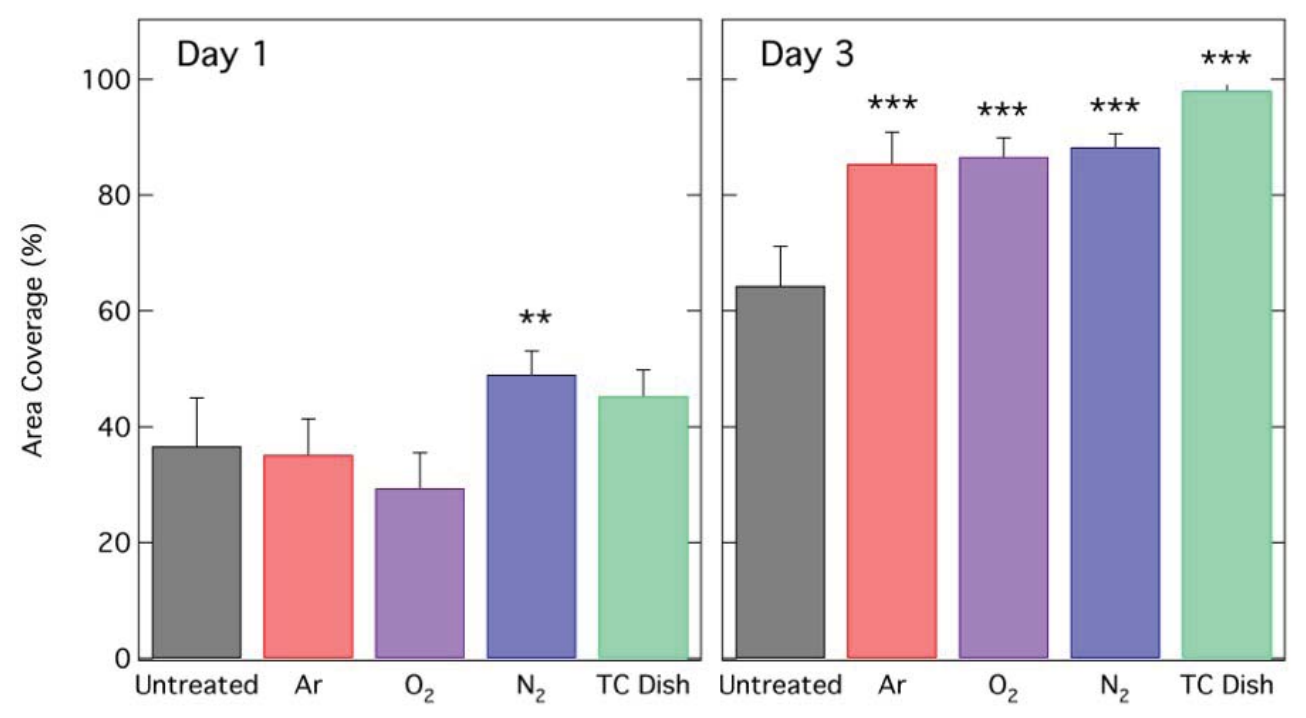

FIG. 6: Cell adhesion on plasma-treated PI substrates measured as percentage of area coverage after 1 and $3 \mathrm{~d}$ of culture. Cells were stained with calcein and the percentage of area coverage was determined using ImageJ. Values are reported as mean \pm standard deviation (SD). TC, Tissue culture. $* * p \leq 0.01$ and $* * * p \leq 0.001$ statistical significance, with one-way analysis of variance (ANOVA) and Dunnett's post-test.

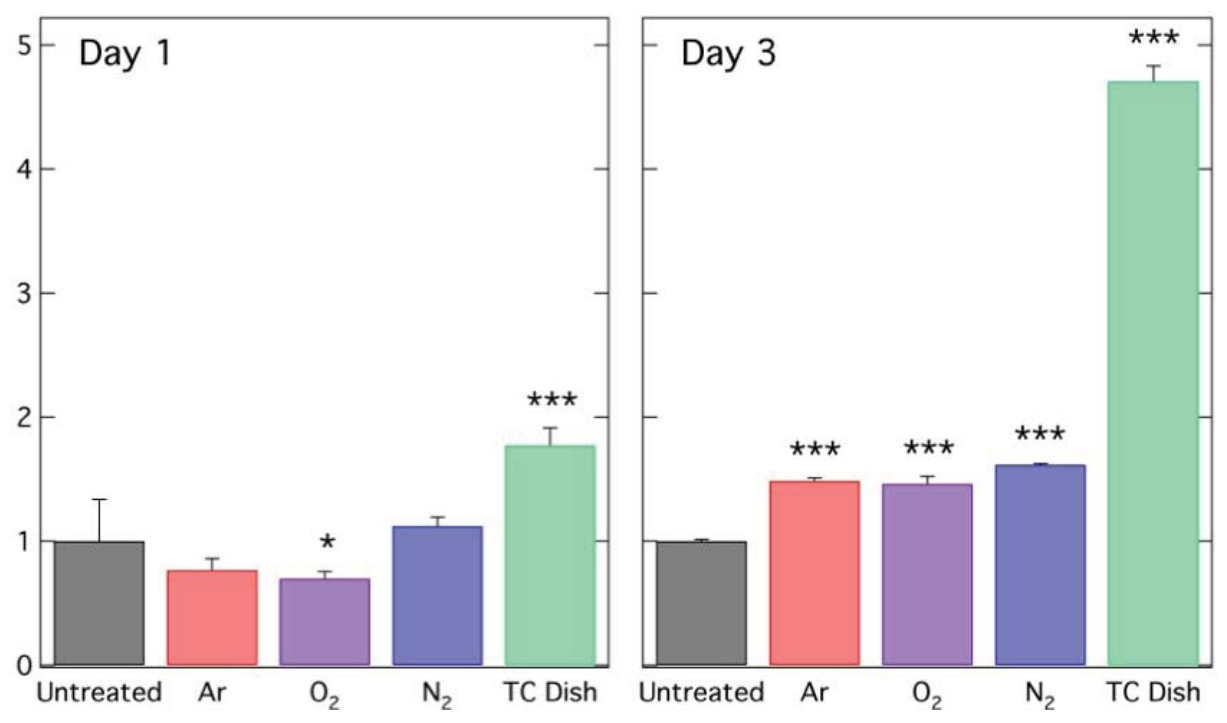

FIG. 7: Cell proliferation on plasma-treated PI substrates normalized with untreated PI after 1 and $3 \mathrm{~d}$ of culture. Cell proliferation was measured using the PrestoBlue cell-viability assay. Values are reported as mean \pm SD. TC, Tissue culture. $* p \leq 0.05$ and $* * * p \leq 0.001$ statistical significance, with one-way analysis of variance (ANOVA) and Dunnett's post-test. 
a result of the inert nature of the Ar gas, whereas $\mathrm{O}_{2}$-treated surfaces will attach hydroxyl groups on the surface. In general, the improved biocompatibility properties of plasmatreated PI substrates at day 3 may also be attributed to the increase in hydrophilicity of the PI surfaces through morphological modifications and changes in SFE brought about by plasma treatment. ${ }^{19,20}$

\section{CONCLUSIONS}

Improving directed cell responses to biofunctionalized interfaces requires the ability to tune surface properties for desired cell adhesion and subsequent proliferation. Plasmatreated PI surfaces exhibited improvements in terms of cell attachment as well as proliferation in comparison to untreated surfaces. Among the three gas species used, $\mathrm{N}_{2}$ plasmatreated surfaces showed strong affinity to biomolecules, especially during the first day of cell seeding, compared to that of untreated samples. This may be attributed to the possible nitrogen-containing functional groups on the surface that further enhance cell adhesion and proliferation. Surface functionalization using $\mathrm{N}_{2}$ plasma must be conducted to further clarify the mechanisms of action between the cell and polymer substrate interactions.

\section{ACKNOWFDGENIS}

M.R.V. is grateful for the support of the Balik- $\mathrm{PhD}$ research grant OVPAA-BPhD2014-01. E.I.P. acknowledges support of the Balik-PhD research grant OVPAA-BPhD2015-03.

\section{REFERENCES}

1. Park WJ, Yoon SG, Jung WS, Yoon DH. Effect of dielectric barrier discharge on surface modification characteristics of polyimide film. Surf Coat Technol. 2007;201:5017-20.

2. Zuo M, Takeichi T, Matsumoto A, Tsutsumi K. Surface characterization of polyimide films. Colloid Polym Sci. 276;1998:555-64.

3. Chen H, Iliev MN, Liu JR, Ma KB, Chu WK, Badi N, Bensaoula A, Svedberg EB. Room-temperature deposition of diamond-like carbon field emitter on flexible substrates. Nucl Instrum Methods Phys Res B. 2006;243:75-8.

4. Tanemura M, Hatano H, Kitazawa M, Tanaka J, Okita T, Lau SP, Yang HY, Yu SF, Huang L, Miao L, Tanemura S. Room-temperature growth of carbon nanofibers on plastic substrates. Surf Sci. 2006;600:3663-7.

5. Richardson RR, Miller JA, Reichert WM. Polyimides as biomaterials: Preliminary biocompatibility testing. Biomaterials. 1993;14:627-35.

6. Righi M, Bossi S, Puleo GL, Guidetti G, Wieringa P, Cutrone A, Micera S. Surface modification of polyimide thin films for peripheral invasive neural interfaces. J Med Devices. 2013;7(2):020937.

7. Van Vlierberghe S, Sirova M, Rossman P, Thielecke H, Boterberg V, Rihova B, Schacht E, Dubruel P. Surface modification of polyimide sheets for regenerative medicine applications. Biomacromolecules. 2010;11:2731-9.

8. Maenosono H, Saito H, Nishioka Y. A transparent polyimide film as a biological cell culture sheet with microstructures. J Biomater Nanobiotechnol. 2014;5(1):41908.

9. Park G, Chung HJ, Kim K, Lim SA, Kim J, Kim YS, Liu Y, Yeo WH, Kim RH, Kim SS, Kim JS, Jung

Volume 8, Issue 1, 2018 
YH, Kim TI, Yee C, Rogers JA, Lee KM. Immunologic and tissue biocompatibility of flexible/stretchable electronics and optoelectronics. Adv Healthcare Mater. 2014;3(4):515-25.

10. Chu PK, Chen JY, Wang LP, Huang N. Plasma-surface modification of biomaterials. Mater Sci Eng R. 2002;36:143-206.

11. Siow KS, Britcher L, Kumar S, Griesser H. Plasma methods for the generation of chemically reactive surfaces for biomolecule immobilization and cell colonization: A review. Plasma Proc Polym. 2006;3:392-418.

12. Oehr C. Plasma surface modification of polymers for biomedical use. Nucl Instr Meth Phys Res B. 2003;208:40-7.

13. Liston EM, Martinu L, Wertheimer MR. Plasma surface modification of polymers for improved adhesion: A critical review. J Adhesion Sci Technol. 1993;7:1091-127.

14. Cagomoc CMD, Vasquez MR. Enhanced chromium adsorption capacity via plasma modification of natural zeolites. Jpn J Appl Phys. 2017;56(1S):01AF02.

15. Owens DK, Wendt RC. Estimation of the surface free energy of polymers. J Appl Polym. 1969;13: 1741-7.

16. Schneider CA, Rasband WS, Eliceiri KW. NIH image to ImageJ: 25 years of image analysis. Nat Methods. 2012;9:671-5.

17. Arima $\mathrm{Y}$, Iwata $\mathrm{H}$. Effect of wettability and surface functional groups on protein adsorption and cell adhesion using well-defined mixed self-assembled monolayers. Biomaterials. 2007;28:3074-82.

18. Lee JH, Jung HW, Kang IK, Lee HB. Cell behaviour on polymer surfaces with different functional groups. Biomaterials. 1994;15:705-11.

19. Schakenraad JM, Busscher HJ. Cell-polymer interactions: The influence of protein adsorption. Colloids Surf. 1989;42:331-43.

20. van der Valk P, van Pelt AW, Busscher HJ, de Jong HP, Wildevuur CR, Arends J. Interaction of fibroblasts and polymer surfaces: Relationship between surface free energy and fibroblast spreading. J Biomed Mater Res. 1983;17:807-17. 